\title{
Von Neumann Uniqueness Theorem doesn't hold in Hyperbolic Quantum Mechanics
}

Andrei Khrennikov, Gavriel Segre丹

International Center for Mathematical Modelling in Physics and Cognitive Sciences, University of Växjö, S-35195, Sweden

It is shown that Von Neumann Uniqueness Theorem doesn't hold in Hyperbolic Quantum Mechanics

*Electronic address: Andrei.Khrennikov@msi.vxu.se, Gavriel.Segre@msi.vxu.se 
Contents

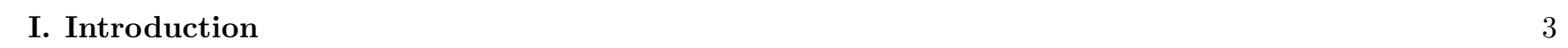

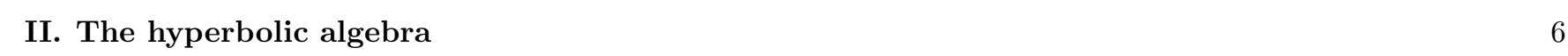

$\begin{array}{ll}\text { III. Hvperbolic Hilbert Spaces } & 7\end{array}$

\begin{tabular}{ll}
\hline IV. Hvperbolic Quantum Mechanics & 11
\end{tabular}

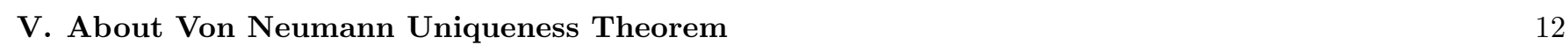

VI. Position and momentum representations of the Hvperbolic Canonical Commutation Relation 15

\begin{tabular}{|l|l|}
\hline A. Hyperbolic functions at rapid decrease and hyperbolic tempered distributions & 17
\end{tabular}

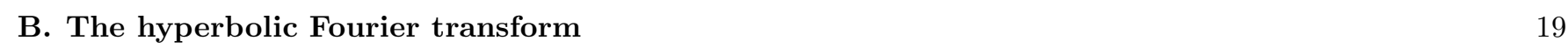

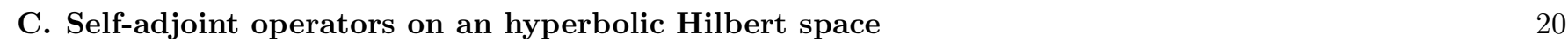

References 23 


\section{INTRODUCTION}

Following Adler's [1] formalization of Feynman's basic observations [2] concerning quantum probabilities, let us recall that a key feature of quantum probabilities consists in that they don't obey the usual formula of probabilities' composition:

$$
P_{c a}=\sum_{b} P_{c b} \cdot P_{b a}
$$

but a formula for probabilities amplitudes's composition:

$$
\Phi_{c a}=\sum_{b} \Phi_{c b} \cdot \Phi_{b a}
$$

where the probabilities amplitudes $\Phi$ 's take value on a finite dimensional algebra A over $\mathbb{R}[3]$ on which a modulus function $N: A \mapsto \mathbb{R}$ is defined such that:

$$
\begin{aligned}
& P_{b a}=N^{2}\left(\Phi_{b a}\right) \\
& P_{c b}=N^{2}\left(\Phi_{c b}\right) \\
& P_{c a}=N^{2}\left(\Phi_{c a}\right)
\end{aligned}
$$

where both the algebra $\mathrm{A}$ and the modulus function has to be determined imposing reasonable physical and mathematical constraints.

From a mathematical side it is natural to require that $\mathrm{N}$ is a norm over $\mathrm{A}$.

From a physical side the imposition of the Correspondence Principle requires that, in the absence of quantum interference effects, probability amplitude superposition (i.e. eq. 1.2) should reduce to probability superposition (i.e. eq. 1.1). This leads (cfr. [1] for details) to the condition that the norm $\mathrm{N}$ has to be multiplicative.

One has that:

\section{Theorem I.1}

\section{ALBERT'S THEOREM}

HP:

A finite dimensional algebra with unit over $\mathbb{R}$

$$
\mathrm{N} \text { multiplicative norm over } \mathrm{A}
$$

TH:

$$
A \in\{\mathbb{R}, \mathbb{C}, \mathbb{H}, \mathbb{O}\}
$$

where $\mathbb{H}$ is the (noncommutative) algebra of Hamilton's quaternions and $\mathbb{O}$ is the (noncommutative and nonassociative) algebra of Cayley's octonions whose definition we briefly recall.

The generic element of an $(n+1)$-dimensional algebra with unit A may be expressed as:

$$
\Phi=\sum_{i=0}^{n} r_{i} e_{i}
$$

where $e_{0}=1, \cdots, e_{n}$ are the basis elements of the algebra obeying multiplication law:

$$
e_{i} \cdot e_{j}=\sum_{k=0}^{n} f_{i j k} e_{k} \quad i, j=0, \cdots, n
$$

with the real-valued structure constant $f_{i j k}$ 's obeying the following constraints:

$$
f_{0 i j}=\delta_{i j} i, j=0, \cdots, n
$$




$$
f_{i 0 j}=\delta_{i j} \quad i, j=0, \cdots, n
$$

that may be immediately derived imposing that:

$$
e_{i} \cdot e_{0}=e_{0} \cdot e_{i}=e_{0} i=0, \cdots, n
$$

The algebra of quaternions $\mathbb{H}$ corresponds to the case $n=3$ and:

$$
e_{i} \cdot e_{j}=-\delta_{i j}+\sum_{k=1}^{3} \epsilon_{i j k} e_{k}
$$

where $\epsilon_{i j k}$ is the Levi Civita's tensor, i.e. the totally antisymmetric tensor with $\epsilon_{123}=1$.

A multiplicative norm on $\mathbb{H}$ is given by:

$$
N\left(\sum_{i=0}^{3} r_{i} e_{i}\right):=\sqrt{\sum_{i=0}^{3} r_{i}^{2}}
$$

The algebra of octonions $(\mathbb{O}$ corresponds to the case $n=7$ and:

$$
e_{i} \cdot e_{j}=-\delta_{i j}+\sum_{k=1}^{3} \gamma_{i j k} e_{k}
$$

where $\gamma_{i j k}$ is the totally antisymmetric tensor such that:

$$
\gamma_{123}=\gamma_{246}=\gamma_{435}=\gamma_{367}=\gamma_{651}=\gamma_{572}=\gamma_{714}=1
$$

A multiplicative norm on $\mathbb{O}$ is given by:

$$
N\left(\sum_{i=0}^{7} r_{i} e_{i}\right):=\sqrt{\sum_{i=0}^{7} r_{i}^{2}}
$$

Let us observe, anyway, that from a physical viewpoint it seems rather natural to require that:

$$
\Phi_{a b} \cdot \Phi_{b c}=\Phi_{b c} \cdot \Phi_{a b}
$$

that leads to the constraint that $\mathrm{A}$ has to be commutative; so, by Albert Theorem, $A \in\{\mathbb{R}, \mathbb{C}\}$.

Since $\mathbb{R}$ is to strict to allow all the superpositions of states observed experimentally, it follows that Nature has chosen $A=\mathbb{C}$.

Let us now observe that, with the exception of the non-associative octonions' algebra $\mathbb{O}$, the algebras allowed by Albert's Theorem are finite-dimensional real Clifford algebras [4]:

$$
\begin{aligned}
\mathbb{R} & =C l_{0,0} \\
\mathbb{C} & =C l_{0,1} \\
\mathbb{H} & =C l_{0,2}
\end{aligned}
$$

To understand better the structural properties of the choice of $C l_{0,1}$ made by Nature, it may be interesting to investigate how the mathematical structure of Quantum Mechanics is modified by the ansatz:

$$
C l_{0,1} \mapsto C l_{1,0}
$$

corresponding, in terms of the structure constants, to the ansatz:

$$
f_{111}=0 f_{110}=1 \mapsto f_{111}=0 f_{110}=-1
$$


Since, as we have shown in [5], there are many reasons to call $\mathbb{G}:=C l_{1,0}$ the hyperbolic algebra, we will denote the $C l_{1,0^{-}}$Quantum Mechanics as Hyperbolic Quantum Mechanics.

Such a mathematical theory emerged in the research of one of the authors [ [] , 7], [8] and of other scientists (see [9], 10], 11], 12], 13], 14], 15] and references therein).

Let us observe, first of all, that the modulus function:

$$
N\left(\sum_{i=0}^{1} r_{i} e_{i}\right):=\sqrt{\sum_{i=0}^{1} r_{i}^{2}}
$$

is a norm, though not multiplicative.

\section{Remark I.1}

We would like to stress, from the physical point of view, that we are in no way claiming that Quantum Mechanics, as a physical theory, is wrong or has to be modified.

We are simply analyzing an alternative mathematical theory whose structure could allow to get some insight of the $C l_{0,1}$-choice made by Nature 


\section{THE HYPERBOLIC ALGEBRA}

Let us define the hyperbolic algebra as the ring $\mathbb{G}$ of numbers of the form $x+j y$, where $x, y \in \mathbb{R}$ while $\mathrm{j}$, called the hyperbolic imaginary unit, is such that $j^{2}=+1$.

The elements of such an algebra has been called in the mathematical literature with different names (cfr. 16 ] and references therein): hyperbolic numbers, double numbers, split complex numbers, perplex numbers, and duplex numbers.

We will call them hyperbolic numbers and we will refer to $\mathrm{j}$ as to the hyperbolic imaginary unit.

The complex field $\mathbb{C}$ and the hyperbolic ring $\mathbb{G}$ are the two bidimensional Clifford algebras $[\underline{5}$ :

$$
\begin{aligned}
& \mathbb{C}=C l_{0,1} \\
& \mathbb{G}=C l_{1,0}
\end{aligned}
$$

Given $z=x+j y \in \mathbb{G}$ :

\section{Definition II.1}

CONJUGATE OF z:

$$
\bar{z}:=x-j y
$$

\section{Definition II.2}

NORM OF z:

$$
\|z\|:=\sqrt{x^{2}+y^{2}}
$$

\section{Definition II.3}

\section{LIGHT CONE OF $z$ :}

$$
V_{z}:=\left\{z^{\prime}=x^{\prime}+j y^{\prime} \in \mathbb{G}: y^{\prime}= \pm\left(x^{\prime}-x\right)+y\right\}
$$

One has that:

\section{Theorem II.1}

- $\mathbb{G}$ is a commutative ring

- $\mathbb{G}$ is not a field

\section{PROOF:}

1. by definition the addition and the multiplication in $\mathbb{G}$ are commutative and associative, the multiplication is distributive with respect to the addition, there exists a null element 0 with respect to addition, there exists an identity element 1 with respect to multiplication and every element of $\mathbb{G}$ has an additive inverse

2. given $z=x+j y \in \mathbb{G}$ one has formally that:

$$
\frac{1}{z}=\frac{1}{x+j y}=\frac{x}{x^{2}-y^{2}}-j \frac{y}{x^{2}-y^{2}}
$$

and hence:

$$
\exists z^{-1} \Leftrightarrow z \notin V_{0}
$$

So not every nonzero element of $\mathbb{G}$ has a multiplicative inverse and hence $\mathbb{G}$ is not a field

One has clearly that:

\section{Proposition II.1}

$$
\begin{gathered}
\|z\|^{2} \neq z \bar{z} \forall z \in \mathbb{G}-\mathbb{R} \\
\text { PROOF: }
\end{gathered}
$$

Given $z=x+j y \in \mathbb{G}-\mathbb{R}$ one has that

$$
\|z\|^{2}=x^{2}+y^{2} \neq z \bar{z}=x^{2}-y^{2}
$$




\section{HYPERBOLIC HILBERT SPACES}

Let us start introducing the following:

\section{Definition III.1}

HYPERBOLIC LINEAR SPACE:

a triple $(V,+, \cdot)$ where $\mathrm{V}$ is a set while $+: V \times V \mapsto V$ and $\cdot: \mathbb{G} \times V \mapsto V$ are such that:

$$
\begin{gathered}
u+v=v+u \quad \forall u, v \in V \\
(u+v)+w=u+(v+w) \quad \forall u, v, w \in V \\
\exists 0 \in V: u+0=u \quad \forall u \in V \\
\lambda(u+v)=\lambda u+\lambda v \quad \forall u, v \in V, \forall \lambda \in \mathbb{G} \\
(\lambda+\mu) u=\lambda u+\mu u \quad \forall u, \in V, \forall \lambda, \mu \in \mathbb{G} \\
1 u=u \quad \forall u \in V
\end{gathered}
$$

We can then introduce the following:

\section{Definition III.2}

HYPERBOLIC INNER PRODUCT SPACE:

a quatruple $(V,+, \cdot,(\cdot, \cdot))$ such that:

- $(V,+, \cdot)$ is an hyperbolic linear space

- $(\cdot, \cdot): V \times V \mapsto \mathbb{G}$ is such that:

$$
\begin{gathered}
(u, v+w)=(u, v)+(u, w) \forall u, v, w \in V \\
(u, \lambda v)=\lambda(u, v) \forall u, v \in V, \forall \lambda \in \mathbb{G} \\
(u, v)=\overline{(v, u)} \forall u, v \in V
\end{gathered}
$$

\section{Example III.1}

Let $\mathbb{G}^{n}$ denote the set of all n-ples of hyperbolic numbers; given $x=\left(x_{1}, \cdots, x_{n}\right), y=\left(y_{1}, \cdots, y_{n}\right) \in \mathbb{G}^{n}$ define:

$$
(x, y):=\sum_{i=1}^{n} \bar{x}_{i} y_{i}
$$

$\left(\mathbb{G}^{n},(\cdot, \cdot)\right)$ is then an hyperbolic inner product space

Given an hyperbolic inner-product space $(V,+, \cdot,(\cdot, \cdot))$ and a linear operator $U: V \mapsto V$ :

\section{Definition III.3}

U IS UNITARY:

$$
(U x, U y)=(x, y) \forall x, y \in V
$$

\section{Definition III.4}


HYPERBOLIC NORMED LINEAR SPACE:

a couple $(V,\|\cdot\|)$ where:

- $\mathrm{V}$ is an hyperbolic linear space

- $\|\cdot\|$ is a map $\|\cdot\|: \mathbb{G} \mapsto \mathbb{R}$ such that:

$$
\begin{gathered}
\|v\| \geq 0 \quad \forall v \in V \\
\|v\|=0 \Leftrightarrow v=0 \\
\exists c \in \mathbb{R}_{+}:(\|\alpha v\| \leq c\|\alpha\|\|v\| \forall v \in V, \forall \alpha \in \mathbb{G}) \\
\|u+v\| \leq\|u\|+\|v\| \quad \forall u, v \in V
\end{gathered}
$$

Given two hyperbolic normed linear spaces $\left(V_{1},\|\cdot\|_{1}\right)$ and $\left(V_{2},\|\cdot\|_{2}\right)$ and a linear operator $T: V_{1} \mapsto V_{2}$ :

\section{Definition III.5}

T IS BOUNDED:

$$
\|T\|:=\sup _{\|v\|_{1}=1}\|T v\|_{2} \in \mathbb{R}
$$

\section{Definition III.6}

\section{HYPERBOLIC BANACH SPACE}

an hyperbolic normed linear space $(V,\|\cdot\|)$ which is complete as a metric space in the induced metric $d(u, v):=$ $\|u-v\|$.

\section{Definition III.7}

HYPERBOLIC HILBERT SPACE

a triple $(V,(\cdot, \cdot),\|\cdot\|)$ such that:

- $(V,(\cdot, \cdot))$ is an hyperbolic inner-product space

- $(V,\|\cdot\|)$ is an hyperbolic Banach space

$$
\exists c \in \mathbb{R}_{+}:(\|(u, v)\| \leq c\|u\|\|v\| \forall u, v \in V)
$$

\section{Remark III.1}

Let us observe that all the introduced notions of the form "hyperbolic $\mathrm{x}$ ", with $\mathrm{x}=$ linear space, normed linear space, Banach space, Hilbert space, has not to be intended as particular cases of the respective notion x:

since $\mathbb{G}$ is not a field, an hyperbolic linear space is not a linear space but only a module over the ring $\mathbb{G}$ and so on.

\section{Example III.2}

Given the hyperbolic inner product space $\left(\mathbb{G}^{n},(\cdot, \cdot)\right)$ let us introduce the hyperbolic Banach space $\left(\mathbb{G}^{n},\|\cdot\|\right)$ defined as:

$$
\left\|\left(x_{1}+j y_{1}, \cdots, x_{n}+j y_{n}\right)\right\|:=\sqrt{\sum_{i=1}^{n}\left(x_{i}^{2}+y_{i}^{2}\right)}
$$


Given $x=\left(a_{1}+j b_{1}, \cdots, a_{n}+j b_{n}\right) \in \mathbb{G}^{n}$ and $\alpha=c+j d \in \mathbb{G}$ one has that:

$$
\begin{gathered}
\|\alpha x\|=\sqrt{\sum_{i=1}^{n}\left(c^{2} a_{i}^{2}+c^{2} b_{i}^{2}+d^{2} a_{i}^{2}+d^{2} b_{i}^{2}+4 a_{i} b_{i} c d\right)} \\
\|\alpha\|\|x\|=\sqrt{\sum_{i=1}^{n}\left(c^{2} a_{i}^{2}+c^{2} b_{i}^{2}+d^{2} a_{i}^{2}+d^{2} b_{i}^{2}\right)}
\end{gathered}
$$

Since:

$$
\begin{aligned}
& 2 a_{i} b_{i} c d \leq c^{2} a_{i}^{2}+d^{2} b_{i}^{2} \\
& 2 a_{i} b_{i} c d \leq c^{2} b_{i}^{2}+d^{2} a_{i}^{2}
\end{aligned}
$$

it follows that:

$$
\|\alpha x\| \leq \sqrt{2}\|\alpha\|\|x\|
$$

Furthermore, given $x=\left(a_{1}+j b_{1}, \cdots, a_{n}+j b_{n}\right), y=\left(c_{1}+j d_{1}, \cdots, c_{n}+j d_{n}\right) \in \mathbb{G}^{n}$ one has that:

$$
\begin{gathered}
\|(x, y)\|=\sqrt{\sum_{i=1}^{n}\left(a_{i}^{2} c_{i}^{2}+a_{i}^{2} d_{i}^{2}+b_{i}^{2} c_{i}^{2}+b_{i}^{2} d_{i}^{2}-4 a_{i} b_{i} c_{i} d_{i}\right)} \\
\|x\|\|y\|=\sqrt{\sum_{i=1}^{n}\left(a_{i}^{2} c_{i}^{2}+a_{i}^{2} d_{i}^{2}+b_{i}^{2} c_{i}^{2}+b_{i}^{2} d_{i}^{2}\right)}
\end{gathered}
$$

Since:

$$
\begin{aligned}
& -2 a_{i} b_{i} c_{i} d_{i} \leq a_{i}^{2} c_{i}^{2}+b_{i}^{2} d_{i}^{2} \\
& -2 a_{i} b_{i} c_{i} d_{i} \leq a_{i}^{2} d_{i}^{2}+b_{i}^{2} c_{i}^{2}
\end{aligned}
$$

it follows that:

$$
\|(x, y)\| \leq \sqrt{2}\|x\|\|y\|
$$

$\left(\mathbb{G}^{n},(\cdot, \cdot),\|\cdot\|\right)$ is then an hyperbolic Hilbert space.

\section{Example III.3}

Define $L^{2}(\mathbb{R}, \mathbb{G})$ to be the set of hyperbolic valued measurable functions on $\mathbb{R}$ that satisfy $\int_{-\infty}^{+\infty} d x\|f(x)\|^{2}<+\infty$. Let us introduce:

$$
(f, g):=\int_{-\infty}^{+\infty} d x \bar{f}(x) g(x)
$$

and:

$$
\|\psi\|:=\sqrt{\int_{-\infty}^{+\infty} d x\|\psi(x)\|^{2}}
$$


One has that:

$$
\|z \psi\|=\sqrt{\int_{-\infty}^{+\infty} d x\|z \psi(x)\|^{2}} \leq \sqrt{2} \sqrt{\int_{-\infty}^{+\infty} d x\|z\|^{2}\|\psi(x)\|^{2}}=\sqrt{2}\|z\|\|\psi\| \forall z \in \mathbb{G}, \forall \psi \in L^{2}(\mathbb{R}, \mathbb{G})
$$

Furthermore one has that:

$$
\begin{aligned}
\|(f, g)\|=\left\|\int_{-\infty}^{+\infty} d x \bar{f}(x) g(x)\right\| & \leq \int_{-\infty}^{+\infty} d x\|\bar{f}(x) g(x)\| \leq \sqrt{2} \int_{-\infty}^{+\infty} d x\|\bar{f}(x)\|\|g(x)\| \leq \\
& \leq \sqrt{2} \sqrt{\int_{-\infty}^{+\infty} d x\|f(x)\|^{2}} \sqrt{\int_{-\infty}^{+\infty} d x\|g(x)\|^{2}}=\sqrt{2}\|f\|\|g\| \forall f, g \in L^{2}(\mathbb{R}, \mathbb{G})
\end{aligned}
$$

from which, using the fact that absolute convergence implies convergence, it follows that:

$$
(f, g) \in \mathbb{G} \forall f, g \in L^{2}(\mathbb{R}, \mathbb{G})
$$

$\left(L^{2}(\mathbb{R}, \mathbb{G}),(\cdot, \cdot),\|\cdot\|\right)$ is then an hyperbolic Hilbert space.

As to unbounded operators over an hyperbolic Hilbert space $\mathcal{H}$ let us observe that, as in the analogous case of unbounded operators over a (complex) Hilbert space [17, they will be usually defined only on a dense linear subspace of $\mathcal{H}$. 


\section{HYPERBOLIC QUANTUM MECHANICS}

Hyperbolic numbers emerged in the research of one of the authors [8], [6], 7] as the underlying number system of a mathematical theory, "Hyperbolic Quantum Mechanics" formalized by the following axioms:

\section{AXIOM IV.1}

The pure states of an hyperbolic quantum systems are rays on an hyperbolic Hilbert space $\mathcal{H}$

\section{AXIOM IV.2}

Hyperbolic quantum mechanical observables are linear operators on $\mathcal{H}$ having real spectrum. The expected value of the hyperbolic observable $\hat{O}$ in a state $\psi \in \mathcal{H}$ such that $(\psi, \psi) \neq 0$ is given by:

$$
E_{\psi}(O)=\frac{(\psi, O \psi)}{(\psi, \psi)}
$$

\section{AXIOM IV.3}

The evolution of a pure state $\psi_{0} \in \mathcal{H}$ is described by the hyperbolic analogue of Schrödinger's equation:

$$
j \frac{d \psi(t)}{d t}=H \psi(t), \psi(0)=\psi_{0}
$$




\section{ABOUT VON NEUMANN UNIQUENESS THEOREM}

Let us leave aside for a moment Hyperbolic Quantum Mechanics and let us analyze the status of Von Neumann Uniqueness Theorem in ordinary (complex) Quantum Mechanics.

Given an Hilbert space $\mathcal{H}$, a dense linear subspace $\mathcal{D}$ of $\mathcal{H}$ and two linear operators $\hat{\tilde{Q}}, \hat{\tilde{P}}$ over $\mathcal{H}$ we will say that:

\section{Definition V.1}

$\hat{\tilde{Q}}$ AND $\hat{\tilde{P}}$ ARE A REPRESENTATION OF THE CANONICAL COMMUTATION RELATION OVER $\mathcal{D}$ :

$$
\mathcal{D} \subseteq D(\hat{\tilde{Q}}) \cap D(\hat{\tilde{P}}), \hat{\tilde{Q}} \mathcal{D} \subseteq \mathcal{D}, \hat{\tilde{P}} \mathcal{D} \subseteq \mathcal{D}
$$

$\cdot$

$$
([\hat{\tilde{Q}}, \hat{\tilde{P}}])[\psi]:=\hat{\tilde{Q}}[\psi] \hat{\tilde{P}}[\psi]-\hat{\tilde{P}}[\psi] \hat{\tilde{Q}}[\psi]=i \hat{I}[\psi] \forall \psi \in \mathcal{D}
$$

where $\hat{I}$ is the identity operator over $\mathcal{H}$.

Introduced the following operators on $L^{2}(\mathbb{R}, \mathbb{C})$ :

$$
\begin{gathered}
\left(\hat{Q}_{q} \psi\right)(q):=q \psi(q) \\
\left(\hat{P}_{q} \psi\right)(q):=-i \frac{d \psi(q)}{d q}
\end{gathered}
$$

(where $\mathrm{i}$ is the usual complex imaginary unit such that $i^{2}=-1$ ) defined as the closures of their restriction to the initial domain $\mathcal{S}(\mathbb{R}, \mathbb{C})$, it may be easily verified that $\hat{Q}_{q}$ and $\hat{P}_{q}$ are a representation of the Canonical Commutation Relation called the Schrödinger representation.

Von Neumann Uniqueness Theorem is often expressed in the Physics' literature as the following:

\section{Conjecture V.1}

NAIVE VERSION OF VON NEUMANN UNIQUENESS THEOREM

HP:

$$
\hat{\tilde{Q}} \text { and } \hat{\tilde{P}} \text { are a representation of the Canonical Commutation Relation over the Hilbert space } \mathcal{H}
$$

TH:

$$
\exists \hat{U}: \mathcal{H} \mapsto L^{2}(\mathbb{R}, \mathbb{C}) \text { unitary }: \hat{\tilde{Q}}=\hat{U}^{-1} \hat{Q}_{q} \hat{U} \text { and } \hat{\tilde{P}}=\hat{U}^{-1} \hat{P}_{q} \hat{U}
$$

A mathematically more rigorous investigation allows anyway to infer that [18]:

\section{Theorem V.1}

Conjectur $\nabla .1$ is false

PROOF: 
Let us consider the following Hilbert space $\left(l_{2}(\mathbb{C}),(\cdot, \cdot)\right)$ :

$$
\begin{gathered}
l_{2}(\mathbb{C}):=\left\{\left\{x_{n}\right\}_{n=1}^{\infty}, x_{n} \in \mathbb{C} \forall n: \sum_{n=1}^{\infty}\left|x_{n}\right|^{2}<\infty\right\} \\
\left(\left\{x_{n}\right\}_{n=1}^{\infty},\left\{y_{n}\right\}_{n=1}^{\infty}\right):=\sum_{n=1}^{\infty} \bar{x}_{n} y_{n}
\end{gathered}
$$

its dense linear subspace:

$$
\mathcal{D}:=\left\{\left\{x_{n}\right\}_{n=1}^{\infty} \in l_{2}(\mathbb{C}): \sum_{n=1}^{\infty} x_{n}=0, \text { with only finitely many } x_{n} \neq 0\right\}
$$

Given the infinite-dimensional matrices:

$$
\begin{gathered}
\hat{\tilde{Q}}:=\operatorname{diagonal}(\mathbb{N}) \\
\hat{\tilde{P}}:=-i\left(\begin{array}{ccccc}
0 & -1 & -\frac{1}{2} & -\frac{1}{3} & \cdots \\
1 & 0 & -1 & -\frac{1}{2} & \cdots \\
\frac{1}{2} & 1 & 0 & -1 & \cdots \\
\frac{1}{3} & \frac{1}{2} & 1 & 0 & \cdots \\
\vdots & \vdots & \vdots & \vdots & \ddots
\end{array}\right)
\end{gathered}
$$

one has that $\hat{\tilde{Q}}$ and $\hat{\tilde{P}}$ are a representation over the dense linear subspace $\mathcal{D}$ of the Canonical Commutation Relation unitarily inequivalent to the Schrödinger representation.

For other contra-examples see 19] and references therein

Theorem $\nabla .1$ has led most of the Mathematical Physics' community to consider representations not of the Canonical Commutation Relation but of the following Weyl relation:

$$
\hat{V}_{1}(t) \hat{V}_{2}(s)=\exp (i t s) \hat{V}_{2}(s) \hat{V}_{1}(t) \forall t, s \in \mathbb{R}
$$

of which the strongly continuous unitary groups $\left\{\exp \left(i t \hat{P}_{q}\right)\right\}_{t \in \mathbb{R}}$ and $\left\{\exp \left(i s \hat{Q}_{q}\right)\right\}_{s \in \mathbb{R}}$ are indeed a representation, and to call Von Neumann Uniqueness Theorem the following theorem (for whose proof we demand to [20]):

\section{Theorem V.2}

ON THE UNIQUENESS OF REPRESENTATIONS OF WEYL RELATION:

HP:

$\left\{\hat{V}_{1}(t)\right\}_{t \in \mathbb{R}},\left\{\hat{V}_{2}(s)\right\}_{s \in \mathbb{R}}$ one parameter strongly-continuous unitary group on a separable Hilbert space $\mathcal{H}$ satisfying the Weyl relation

TH:

There are closed linear subspaces $\mathcal{H}_{l}$ such that:

$$
\mathcal{H}=\oplus_{l=1}^{N} \mathcal{H}_{l} \quad N \in \mathbb{N}_{+} \cup\{\infty\}
$$

$$
\hat{U}(t): \mathcal{H}_{l} \mapsto \mathcal{H}_{l}, \hat{V}(s): \mathcal{H}_{l} \mapsto \mathcal{H}_{l} \forall s, t \in \mathbb{R}
$$




$$
\forall l, \exists \hat{T}_{l}: \mathcal{H}_{l} \mapsto L^{2}(\mathbb{R}, \mathbb{C}) \text { unitary }: \hat{T}_{l} \hat{U}(t) \hat{T}_{l}^{-1}=\exp \left(i t \hat{P}_{q}\right) \text { and } \hat{T}_{l} \hat{V}(s) \hat{T}_{l}^{-1}=\exp \left(i s \hat{Q}_{q}\right)
$$

It is anyway possible to insist on working with the Canonical Commutation Relation provided one adds further hypotheses to the Conjecture $\nabla .1$ under which it becomes a theorem.

The first step in this direction is rather trivial, consisting simply in getting rid of the reducibility of representations: given a dense linear subspace $\mathcal{D}$ of $\mathcal{H}$ :

\section{Definition V.2}

$\hat{\tilde{Q}}$ AND $\hat{\tilde{P}}$ ARE A SELF-ADJOINT IRREDUCIBLE REPRESENTATION OF THE CANONICAL COMMUTATION RELATION OVER $\mathcal{D}$

- $\hat{\tilde{Q}}$ and $\hat{\tilde{P}}$ are self-adjoint

- $\hat{\tilde{Q}}$ and $\hat{\tilde{P}}$ are a representation of the Canonical Commutation Relation over $\mathcal{D}$

$$
\nexists \mathcal{I} \text { dense linear subspace of } \mathcal{D} \quad:(\exp (i s \hat{\tilde{Q}}) \mathcal{I} \subseteq \mathcal{I} \forall s \in \mathbb{R}) \text { or }(\exp (i t \hat{\tilde{P}}) \mathcal{I} \subseteq \mathcal{I} \forall t \in \mathbb{R})
$$

Among the many possibilities one is the following [19]:

\section{Theorem V.3}

VON NEUMANN UNIQUENESS THEOREM (IN WEAKENED DIXMIER'S FORM) HP:

$\hat{\tilde{Q}}$ and $\hat{\tilde{P}}$ are a self-adjoint irreducible representation of the Canonical Commutation Relation over a dense linear subspace $\mathcal{D}$ of an Hilbert space $\mathcal{H}$ such that $\hat{\tilde{Q}}$ and $\hat{\tilde{P}}$ are closed and the restriction of $\hat{\tilde{Q}}^{2}+\hat{\tilde{P}}^{2}$ to $\mathcal{D}$ is essentially self-adjoint

TH:

$$
\exists \hat{U}: \mathcal{H} \mapsto L^{2}(\mathbb{R}, \mathbb{C}) \text { unitary }: \hat{\tilde{Q}}=\hat{U}^{-1} \hat{Q}_{q} \hat{U} \text { and } \hat{\tilde{P}}=\hat{U}^{-1} \hat{P}_{q} \hat{U}
$$

The Schrödinger representation of the Canonical Commutation Relation is also called the position representation. Let us now introduce the following operators:

$$
\begin{gathered}
\left(\hat{Q}_{p} \psi\right)(p):=+i \frac{d \psi(p)}{d p} \\
\left(\hat{P}_{p} \psi\right)(p):=p \psi(p)
\end{gathered}
$$

defined as the closures of their restriction to the initial domain $\mathcal{S}(\mathbb{R}, \mathbb{C})$.

It may be easily verified that $\hat{Q}_{p}$ and $\hat{P}_{p}$ are an irreducible self-adjoint representation of the Canonical Commutation Relation, called the momentum representation, over a dense linear subspace of $L^{2}(\mathbb{R}, \mathbb{C})$ over which $\hat{Q}_{p}^{2}+\hat{P}_{p}^{2}$ is essentially self-adjoint.

Applying Theorem $\nabla .3$ it follows that:

\section{Corollary V.1}

ON THE UNITARILY EQUIVALENCE OF THE POSITION AND MOMENTUM REPRESENTATIONS

$$
\exists \hat{U}: \mathcal{H} \mapsto L^{2}(\mathbb{R}, \mathbb{C}) \text { unitary }: \hat{Q}_{p}=\hat{U}^{-1} \hat{Q}_{q} \hat{U} \text { and } \hat{P}_{p}=\hat{U}^{-1} \hat{P}_{q} \hat{U}
$$

Indeed the unitary of Corollary $\nabla .1$ is nothing but the usual (complex) Fourier transform. 


\section{POSITION AND MOMENTUM REPRESENTATIONS OF THE HYPERBOLIC CANONICAL COMMUTATION RELATION}

Given the Hyperbolic Canonical Commutation Relation:

$$
[\hat{q}, \hat{p}]=j \hat{I}
$$

let us consider its position representation in $L^{2}(\mathbb{R}, \mathbb{G})$ :

$$
\begin{gathered}
\left(\hat{q}_{q} \psi\right)(q):=q \psi(q) \\
\left(\hat{p}_{q} \psi\right)(q):=-j \frac{d \psi(q)}{d q}
\end{gathered}
$$

and its momentum representation in $L^{2}(\mathbb{R}, \mathbb{G})$ :

$$
\begin{gathered}
\left(\hat{q}_{p} \psi\right)(p):=+j \frac{d \psi(p)}{d p} \\
\left(\hat{p}_{p} \psi\right)(p):=p \psi(p)
\end{gathered}
$$

where all the operators are defined as the closures of their restriction on the initial domain $\mathcal{S}(\mathbb{R}, \mathbb{G})$.

We will prove the following:

\section{Theorem VI.1}

ON THE UNITARILY INEQUIVALENCE OF THE HYPERBOLIC POSITION AND MOMENTUM REPRESENTATIONS

$$
\nexists \hat{U}: L^{2}(\mathbb{R}, \mathbb{G}) \mapsto L^{2}(\mathbb{R}, \mathbb{G}) \text { unitary : }
$$

$$
\begin{aligned}
& \hat{q}_{p}=\hat{U}^{-1} \hat{q}_{q} \hat{U} \\
& \hat{p}_{p}=\hat{U}^{-1} \hat{p}_{q} \hat{U}
\end{aligned}
$$

PROOF:

Owing to theorem B.1 we know that the required $\hat{U}$ is not the Fourier transform as instead occurs in Quantum Mechanics.

Let us now follow for a moment the non-rigorous Dirac bra-ket formalism.

Starting with:

$$
\begin{gathered}
<q|\hat{q}| \alpha>=q<q \mid \alpha> \\
<q|\hat{p}| \alpha>=-j \frac{d}{d q}<q \mid \alpha>
\end{gathered}
$$

one has in particular that:

$$
<q|\hat{p}| p>=-j \frac{d}{d q}<q \mid p>
$$

and hence:

$$
\left[j \frac{d}{d q}+p\right]<q \mid p>=0
$$


from which it follows that:

$$
<q \mid p>=c \exp (-j p q) \quad c \in \mathbb{G}
$$

But then one has that:

$$
\begin{gathered}
<p|\hat{q}| \alpha>=\int_{-\infty}^{+\infty} d q<p|q><q| \hat{q}\left|\alpha>=\bar{c} \int_{-\infty}^{+\infty} d q \exp (j p q)<q\right| \hat{q} \mid \alpha>= \\
\bar{c} \int_{-\infty}^{+\infty} d q \exp (j p q) q<q\left|\alpha>=\frac{\bar{c}}{j} \frac{d}{d p} \int_{-\infty}^{+\infty} d q \exp (j p q)<q\right| \alpha>= \\
j \frac{d}{d p} \int_{-\infty}^{+\infty} d q<p|q><q| \alpha>=+j \frac{d}{d p}<p \mid \alpha>=\left(\hat{q}_{p} \psi_{\mid \alpha>}\right)(p)
\end{gathered}
$$

where we have used eq6.10 and the completeness condition for position autokets:

$$
\int_{-\infty}^{+\infty} d q|q><q|=\hat{I}
$$

Eq[6.11 implies that:

$$
\begin{aligned}
& \hat{q}_{p}[f]=\left(\mathcal{F} \hat{q}_{q} \mathcal{F}^{-1}\right)[f] \quad \forall f \in N[\mathcal{F}] \\
& \hat{p}_{p}[f]=\left(\mathcal{F} \hat{p}_{q} \mathcal{F}^{-1}\right)[f] \quad \forall f \in N[\mathcal{F}]
\end{aligned}
$$

where:

$$
N[\mathcal{F}]:=\{f \in D(\mathcal{F}): \mathcal{F}[f] \in \mathcal{S}(\mathbb{R}, \mathbb{G})\}
$$

So, assuming ad absurdum the existence of a unitary $\hat{U}: L^{2}(\mathbb{R}, \mathbb{G}) \mapsto L^{2}(\mathbb{R}, \mathbb{G})$ :

$$
\begin{aligned}
& \hat{q}_{p}=\hat{U}^{-1} \hat{q}_{q} \hat{U} \\
& \hat{p}_{p}=\hat{U}^{-1} \hat{p}_{q} \hat{U}
\end{aligned}
$$

, one should have that $\left.\hat{U}\right|_{N[\mathcal{F}]}=\mathcal{F}^{-1}$.

The same formulation of a conjecture claiming the existence an an analogous of Theoren $\nabla .3$ for operators on an hyperbolic Hilbert space would be an highly not trivial task owing to the peculiarities of self-adjoint operators on such a space discussed in the appendix [C]

TheorenVI.1 anyway, automatically implies that such a conjecture would be false, i.e. that Von Neumann Uniqueness Theorem doesn't hold in Hyperbolic Quantum Mechanics.

In fact, if an hyperbolic quantum mechanical analogous of theorem $\overline{V .3}$ existed, it would imply the violation of theorem VI.1 


\section{APPENDIX A: HYPERBOLIC FUNCTIONS AT RAPID DECREASE AND HYPERBOLIC TEMPERED DISTRIBUTIONS}

\section{Definition A.1}

HYPERBOLIC FUNCTIONS OF RAPID DECREASE:

$$
\mathcal{S}(\mathbb{R}, \mathbb{G}):=\left\{f: \mathbb{R} \rightarrow \mathbb{G} \text { infinitely differentiable }:\|f\|_{n, m}:=\sup _{x \in \mathbb{R}}\left\|x^{n} \frac{d^{m}}{d x^{m}} f(x)\right\|<\infty \forall n, m \in \mathbb{N}_{+}\right\}
$$

Let us endow $\mathcal{S}(\mathbb{R}, \mathbb{G})$ with the natural topology induced by the seminorms $\|\cdot\|_{n, m}$.

\section{Definition A.2}

SPACE OF HYPERBOLIC TEMPERED DISTRIBUTIONS:

$$
\mathcal{S}^{\prime}(\mathbb{R}, \mathbb{G}):=\text { topological-dual }[\mathcal{S}(\mathbb{R}, \mathbb{G})]
$$

In particular let us introduce the following:

\section{Definition A.3}

HYPERBOLIC DIRAC DELTA:

$$
\delta \in \mathcal{S}^{\prime}(\mathbb{R}, \mathbb{G}): \delta[f]:=f(0)
$$

Given an hyperbolic tempered distribution $\lambda \in \mathcal{S}^{\prime}(\mathbb{R}, \mathbb{G})$, a family of functions $f_{\alpha}: \mathbb{R} \rightarrow \mathbb{G}$ for every $\alpha \in I:=[a, b]$ with $a, b \in[0,+\infty]$, a measure $\mu$ on $(\mathbb{R}, \mathcal{B}(\mathbb{R}))$ and a number $\bar{\alpha} \in I$ :

\section{Definition A.4}

$f_{\alpha}$ IS A LIMIT-REPRESENTION OF $\lambda$ WITH RESPECT TO $\mu$ FOR $\alpha \rightarrow \bar{\alpha}\left(R E P_{\mu}-\lim _{\alpha \rightarrow \bar{\alpha}} f_{\alpha}=\lambda\right)$

$$
\lim _{\alpha \rightarrow \bar{\alpha}} \int d \mu(x) f_{\alpha}(x) \phi(x)=\lambda[\phi] \forall \phi \in \mathcal{S}(\mathbb{R}, \mathbb{G})
$$

Let us now consider the family of functions:

$$
f_{\alpha}(x):=\int_{-\alpha}^{\alpha} d p \exp (j p x)=\frac{2 \sinh (\alpha x)}{x}
$$

One has that:

Theorem A.1

$$
R E P_{\mu_{\text {Lebesgue }}}-\lim _{\alpha \rightarrow+\infty} f_{\alpha} \neq \delta
$$

$$
\text { PROOF: }
$$

Let us consider the test function $\phi(x):=\exp \left(-x^{2}\right)$.

Since:

$$
\int_{-\alpha}^{\alpha} d p \exp \left(j p x-x^{2}\right)
$$

doesn't converge to $\phi(0)=1$ as $\alpha \rightarrow \infty$ the thesis follows

One has that:

Theorem A.2

EMBEDDING THEOREM

$$
\mathcal{S}(\mathbb{R}, \mathbb{G}) \subset L^{2}(\mathbb{R}, \mathbb{G}) \subset \mathcal{S}^{\prime}(\mathbb{R}, \mathbb{G})
$$


PROOF:

Every function $f \in \mathcal{S}(\mathbb{R}, \mathbb{G})$ can be identified with the functional $f[\cdot] \in \mathcal{S}^{\prime}(\mathbb{R}, \mathbb{G})$ defined as:

$$
f[g]:=\int_{-\infty}^{+\infty} d x g(x) f(x)
$$

From the other side one has that:

$$
\left(\|f\|_{n, m}<+\infty \forall n, m \in \mathbb{N}_{+}\right) \Rightarrow \int_{-\infty}^{+\infty} d x\|f(x)\|^{2} \in(-\infty,+\infty)
$$

and hence $f \in L^{2}(\mathbb{R}, \mathbb{G})$ 


\section{APPENDIX B: THE HYPERBOLIC FOURIER TRANSFORM}

Let us introduce the following:

\section{Definition B.1}

HYPERBOLIC FOURIER TRANSFORM:

the functional $\mathcal{F}: D(\mathcal{F}) \mapsto M A P S(\mathbb{R}, \mathbb{G})$ :

$$
\begin{aligned}
D(\mathcal{F}):= & \left\{f \in \mathcal{S}(\mathbb{R}, \mathbb{G}): \exists \text { finite } \int_{-\infty}^{+\infty} d x \exp (-j p x) f(x)\right\} \\
& (\mathcal{F}[f])(p):=\int_{-\infty}^{+\infty} d x \exp (-j p x) f(x)
\end{aligned}
$$

Let us observe that:

Proposition B.1

$$
\begin{gathered}
\mathcal{F}[D(\mathcal{F})] \nsubseteq L^{2}(\mathbb{R}, \mathbb{G}) \\
\text { PROOF: }
\end{gathered}
$$

Given the function $f(x):=\frac{1}{\sqrt{2 \pi}} \exp \left(-\frac{x^{2}}{2}\right) \in \mathcal{S}(\mathbb{R}, \mathbb{G})$ one has that:

$$
\mathcal{F}[f](p)=\exp \left(\frac{p^{2}}{2}\right) \notin L^{2}(\mathbb{R}, \mathbb{G})
$$

Proposition B.1]implies that:

Theorem B.1

NO HYPERBOLIC PLANCHEREL THEOREM:

$\mathcal{F}$ doesn't extend to a unitary $\hat{U}: L^{2}(\mathbb{R}, \mathbb{G}) \mapsto L^{2}(\mathbb{R}, \mathbb{G})$ 


\section{APPENDIX C: SELF-ADJOINT OPERATORS ON AN HYPERBOLIC HILBERT SPACE}

Let $\left(X,\|\cdot\|_{X}\right)$ and $\left(Y,\|\cdot\|_{Y}\right)$ be hyperbolic Banach spaces. Given a bounded linear operator $T: X \mapsto Y$ let us introduce the following:

\section{Definition C.1}

HYPERBOLIC BANACH SPACE ADJOINT OF T:

the operator $T^{\prime}: Y^{\star} \mapsto X^{\star}$ :

$$
\left(T^{\prime} l\right)(x):=l(T x) l \in Y^{\star}, x \in X
$$

Let us now consider an hyperbolic Hilbert space $(\mathcal{H},(\cdot, \cdot),\|\cdot\|)$. The hyperbolic Banach space adjoint of a bounded linear operator $T: \mathcal{H} \mapsto \mathcal{H}$ is then an operator $T^{\prime}: \mathcal{H}^{\star} \mapsto \mathcal{H}^{\star}$.

Let us now consider the map $C: \mathcal{H} \mapsto \mathcal{H}^{\star}$ which assigns to each $y \in \mathcal{H}$ the linear functional $(y, \cdot) \in \mathcal{H}^{\star}$.

The key difference with respect to the analogous situation on an Hilbert space [17] is that in our case there is no analogue of Riesz Lemma and hence nobody assures us that $\mathrm{C}$ is surjective.

We are thus led to the following:

\section{Definition C.2}

HYPERBOLIC HILBERT SPACE ADJOINT OF T:

the operator $T^{\dagger}: D\left(T^{\dagger}\right) \mapsto \mathcal{H}$ :

$$
T^{\dagger}:=C^{-1} T^{\prime} C
$$

where $D\left(T^{\dagger}\right)$ is the linear subspace of $\mathcal{H}$ where $C^{-1} T^{\prime} C$ is well-defined.

The notion of hyperbolic Hilbert space adjoint can be extended to unbounded operators in the following way:

given an unbounded linear operator $\mathrm{T}$ defined on a dense subspace $\mathrm{D}(\mathrm{T})$ of an hyperbolic Hilbert space $\mathcal{H}$ :

\section{Definition C.3}

HYPERBOLIC HILBERT SPACE ADJOINT OF T:

the operator $T^{\dagger}: D\left(T^{\dagger}\right) \mapsto \mathcal{H}$ :

$$
\begin{gathered}
D\left(T^{\dagger}\right):=\left\{\phi \in \mathcal{H}:\left(\exists ! \eta_{\phi} \in \mathcal{H}:(T \psi, \phi)=\left(\psi, \eta_{\phi}\right) \forall \psi \in D(T)\right)\right\} \\
T^{\dagger} \phi:=\eta_{\phi}
\end{gathered}
$$

\section{Definition C.4}

T IS SELF-ADJOINT:

$$
D(T)=D\left(T^{\dagger}\right) \text { and } T=T^{\dagger}
$$

A standard theorem of Functional Analysis asserts that the spectrum of a self-adjoint operator on an Hilbert space is a subset of the real line [17].

This is no more true as to self-adjoint operators on a hyperbolic Hilbert space as we will show in the simplest case $\mathcal{H}=\mathbb{G}^{2}$.

Let us introduce at this purpose the following useful bijection $T: M_{n}(\mathbb{G}) \mapsto M_{n}(\mathbb{C})$ :

$$
T\left(\left\{x_{i, j}+j y_{i, j}\right\}_{i, j=1}^{n}\right):=\left\{x_{i, j}+i y_{i, j}\right\}_{i, j=1}^{n}
$$

Given:

$$
A:=\left(\begin{array}{ll}
x_{11}+j y_{11} & x_{12}+j y_{12} \\
x_{21}+j y_{21} & x_{22}+j y_{22}
\end{array}\right) \in M_{2}(\mathbb{G})
$$

one has that:

$$
A^{\dagger}=\bar{A}^{t}=\left(\begin{array}{ll}
x_{11}-j y_{11} & x_{21}-j y_{21} \\
x_{12}-j y_{12} & x_{22}-j y_{22}
\end{array}\right)
$$


Let us introduce the set of self-adjoint matrices:

$$
S A_{2}(\mathbb{G}):=\left\{A \in M_{2}(\mathbb{G}): A^{\dagger}=A\right\}
$$

Clearly one has that:

$$
A \in S A_{2}(\mathbb{G}) \Leftrightarrow y_{11}=0 \text { and } y_{22}=0 \text { and } x_{12}=x_{21} \text { and } y_{12}=-y_{21}
$$

so that the generic matrix $A \in S A_{2}(\mathbb{G})$ is of the form:

$$
A=\left(\begin{array}{cc}
x_{11} & x_{12}+j y_{12} \\
x_{12}-j y_{12} & x_{22}
\end{array}\right)
$$

Let us compare the eigenvalue equation of $\mathrm{A}$ and $\mathrm{T}(\mathrm{A})$. For the latter the equation:

$$
\operatorname{det}(T(A)-\lambda I)=0
$$

has solution:

$$
\lambda=\frac{x_{11}+x_{22} \pm \sqrt{\left(x_{11}-x_{22}\right)^{2}+4 x_{12}^{2}+4 y_{12}^{2}}}{2}
$$

and since the discriminant $\Delta:=\left(x_{11}-x_{22}\right)^{2}+4 x_{12}^{2}+4 y_{12}^{2} \geq 0$ :

- if $\Delta:=\left(x_{11}-x_{22}\right)^{2}+4 x_{12}^{2}+4 y_{12}^{2}>0$ then $\mathrm{T}(\mathrm{A})$ has 2 real eigenvalues:

$$
\begin{aligned}
& \lambda_{1}=\frac{x_{11}+x_{22}+\sqrt{\Delta}}{2} \in \mathbb{R} \\
& \lambda_{2}=\frac{x_{11}+x_{22}-\sqrt{\Delta}}{2} \in \mathbb{R}
\end{aligned}
$$

- if $\Delta:=\left(x_{11}-x_{22}\right)^{2}+4 x_{12}^{2}+4 y_{12}^{2}=0$ then $\mathrm{T}(\mathrm{A})$ has 1 real eigenvalue:

$$
\lambda=\frac{x_{11}+x_{22}}{2} \in \mathbb{R}
$$

As to A, instead, the equation:

$$
\operatorname{det}(A-\lambda I)=0
$$

has solution:

$$
\lambda=\frac{x_{11}+x_{22} \pm \sqrt{\left(x_{11}-x_{22}\right)^{2}+4 x_{12}^{2}-4 y_{12}^{2}}}{2}
$$

If follows that:

- if $\Delta:=\left(x_{11}-x_{22}\right)^{2}+4 x_{12}^{2}-4 y_{12}^{2}>0$ then A has 4 eigenvalues of which only two are reals:

$$
\begin{aligned}
& \lambda_{1}=\frac{x_{11}+x_{22}+\sqrt{\Delta}}{2} \in \mathbb{R} \\
& \lambda_{2}=\frac{x_{11}+x_{22}-\sqrt{\Delta}}{2} \in \mathbb{R} \\
& \lambda_{3}=\frac{x_{11}+x_{22}+j \sqrt{\Delta}}{2} \notin \mathbb{R} \\
& \lambda_{4}=\frac{x_{11}+x_{22}-j \sqrt{\Delta}}{2} \notin \mathbb{R}
\end{aligned}
$$


- if $\Delta:=\left(x_{11}-x_{22}\right)^{2}+4 x_{12}^{2}-4 y_{12}^{2}=0$ then $\mathrm{A}$ has 1 real eigenvalue:

$$
\lambda=\frac{x_{11}+x_{22}}{2} \in \mathbb{R}
$$

- if $\Delta:=\left(x_{11}-x_{22}\right)^{2}+4 x_{12}^{2}-4 y_{12}^{2}<0$ then $\mathrm{A}$ has no eigenvalues

In particular we have shown that a matrix $A \in S A_{2}(\mathbb{G})$ cannot be always diagonalized, a fact that by itself proves that the Spectral Theorem doesn't hold for self-adjoint operators on an hyperbolic Hilbert space.

This fact implies that given a self-adjoint operator A on an hyperbolic Hilbert space:

- if $\mathrm{A}$ is bounded, the exponential of $\mathrm{A}$ can be defined by power-series:

$$
\exp (j t A):=\sum_{n=0}^{\infty} \frac{(j t)^{n} A^{n}}{n !}
$$

- if $\mathrm{A}$ is unbounded, not only the exponential of $\mathrm{A}$ cannot be defined by power series (as occurs also for an unbounded operator on a (complex) Hilbert space), but one cannot use the functional calculus form of the Spectral theorem; it follows that no definition of $\exp (j t A)$ can be given in this way.

As a consequence it follows that no analogue exists on an Hyperbolic Hilbert Space of the Stone Theorem that on a (complex) Hilbert space states the existence of a bijection between self-adjoint operators and strongly-continuous unitary groups associating to each self-adjoint operator A the strongly-continuous unitary $\operatorname{group}\{\exp (i t A)\}_{t \in \mathbb{R}}$. 
[1] S.L Adler. Quaternionic Quantum Mechanics and Quantum Fields. Oxford University Press, 1995.

[2] R.P. Feynman A.R. Hibbs. Quantum Mechanics and Path Integrals. Mc Graw Hill, 1965.

[3] I. R. Shafarevich. Basic Notions of Algebra. Springer-Verlag, Berlin, 1997.

[4] K. Gurlebeck W. Sprossig. Quaternionic and Clifford Calculus for Physicists and Engineers. John Wiley and Sons, Baffins Lane, Chichester (England), 1997.

[5] A. Khrennikov G. Segre. An introduction to hyperbolic analysis. math-ph/0507053 2005.

[6] A. Khrennikov. Interference of probabilities and number field structure of quantum models. Annalen der Physik, 12(10):575$585,2003$.

[7] A. Khrennikov. Hyperbolic quantum mechanics. Advances in Applied Clifford Algebras, 13(1):1-9, 2003.

[8] A. Khrennikov. Contextual approach to quantum mechanics and the theory of the fundamental prespace. J. Math. Phys., 45(3):902-921, 2004.

[9] J. Kocik. Duplex Numbers, Diffusion Systems, and Generalized Quantum Mechanics. International Journal of Theoretical Physics, 38(9), 1999.

[10] Y. Xuegang. Hyperbolic Hilbert Space. Advances in Applied Clifford Algebras, 10(1):49-60, 2000.

[11] Z. Zheng Y. Xuegang. Hyperbolic Schrodinger Equation. Advances in Applied Clifford Algebras, 14(2):207-213, 2004.

[12] D. Rochon S. Tremblay. Bicomplex Quantum Mechanics. Advances in Applied Clifford Algebras, 14(2):231-248, 2004.

[13] S. Ulrych. The Poincare' mass operator in terms of a hyperbolic algebra. Physics Letters B, (612):89-91, 2005.

[14] S. Ulrych. Symmetries in the hyperbolic Hilbert space. Physics Letters B, (618):233-236, 2005.

[15] S. Ulrych. Relativistic quantum physics with hyperbolic numbers. Physics Letters B, (625):313-323, 2005.

[16] B. Jancewicz. The Extended Grassmann Algebra of $\mathrm{R}^{3}$. In W.E. Baylis, editor, Clifford (Geometric) Algebras With Applications in Physics, Mathematics and Engineering, pages 389-421. Birkhauser, Boston, 1996.

[17] M. Reed B. Simon. Methods of Modern Mathematical Physics: vol.1 - Functional Analysis. Academic Press, 1980.

[18] W. Thirring. A Course in Mathematical Physics - vol.3: Quantum Mechanics of Atoms and Molecules. Springer-Verlag, Berlin, 1981.

[19] S.J. Summers. On the Stone-von Neumann Uniqueness Theorem and Its Ramifications. In M. Redei N. Stöltzner, editor, John Von Neumann and the Foundations of Quantum Physics, pages 135-152. Kluwer Academic Publisher, Dordrecht, 2001.

[20] M. Reed B. Simon. Methods of Modern Mathematical Physics: vol.3 - Scattering Theory. Academic Press, 1979. 